\title{
Comparação do perfil epidemiológico da tuberculose antes e após a COVID-19 no estado do Pará
}

\author{
Comparison of the epidemiological profile of tuberculosis before and after COVID-19 in the \\ state of Pará
}

Comparación del perfil epidemiológico de la tuberculosis antes y después de la COVID-19 en el estado de Pará

Caio César Amaral Amaral ${ }^{*}$, Débora da Costa Silva ${ }^{1}$, Élri Nayelle Vasconcelos Soares ${ }^{1}$, Jéssica Nayara Espíndola Almeida Maia1, Fabiolla da Silva dos Santos².

\section{RESUMO}

Objetivo: Realizar a comparação do perfil epidemiológico da Tuberculose (TB) no Estado do Pará, fazendo uma comparação da doença em momentos distintos, antes e após o surgimento da Síndrome Respiratória Aguda Grave (SARS-COV-2). Métodos: Trata-se de um estudo observacional descritivo com abordagem quantitativa, realizado pelo levantamento de dados do Sistema de Informações de Agravos de Notificação (SINAN), referente a casos de TB no cenário pré e pós Covid-19 (2016 a 2021). Resultados: Segundo a Organização Mundial da Saúde (OMS) o Brasil consta entre os 20 países com as mais altas incidências por casos absolutos de TB do mundo, o Pará apresenta uma média anual de 4.166 casos de tuberculose, estar relacionado a questões no desenvolvimento social, precariedade na saúde; deficiência da gestão pública, que limitam as ações de prevenção, por consequência, tem-se aumento dos casos da doença. Em 2020, observou-se uma queda de $16 \%$ na notificação de casos novos em comparação a 2019. Esses números podem ser explicados, pelas medidas preventivas adotadas durante a pandemia. Conclusão: $O$ estado do Pará apesar das adversidades sociais e econômicas tem demostrado redução nos índices da TB e do novo coronavírus, segundo os boletins epidemiológicos, isso pode estar ligado as medidas de distanciamento social.

Palavras-chave: Tuberculose, COVID-19, Pandemia, Doença respiratórias.

\begin{abstract}
Objective: To compare the epidemiological profile of Tuberculosis (TB) in the State of Pará, comparing the disease at different times, before and after the onset of Severe Acute Respiratory Syndrome (SARS-COV-2). Methods: This is an observational study descriptive with a quantitative approach, carried out by collecting data from the Notifiable Diseases Information System (SINAN), referring to TB cases in the pre and post Covid-19 scenario (2016 to 2021). Results: According to the World Health Organization (WHO), Brazil is among the 20 countries with the highest incidences of absolute cases of TB in the world, Pará has an annual average of 4,166 cases of tuberculosis, being related to issues in social development, precarious health; deficiency of public management, which limit prevention actions, consequently, there has been an increase in the number of cases of the disease. In 2020, there was a $16 \%$ drop in notification of new cases compared to 2019 . These numbers can be explained by the preventive measures adopted during the pandemic. Conclusion: The state of Pará, despite social and economic adversities, has shown a reduction in the rates of TB and the new coronavirus, according to epidemiological bulletins, this may be linked to measures of social distancing.
\end{abstract}

Keywords: Tuberculosis, COVID-19, Pandemic, Respiratory disease.

\section{RESUMEN}

Objetivo: Comparar el perfil epidemiológico de la Tuberculosis (TB) en el Estado de Pará, comparando la enfermedad en diferentes momentos, antes y después de la aparición del Síndrome Respiratorio Agudo Severo (SARS-COV-2). Métodos: Se trata de un estudio observacional descriptivo con un análisis cuantitativo

${ }^{1}$ Escola Superior da Amazônia (ESAMAZ), Belém - PA. *E-mail: jessica22espindola@gmail.com

2 Universidade Federal do Pará (UFPA), Belém - PA. 
enfoque, realizado a partir de la recolección de datos del Sistema de Información de Enfermedades Notificables (SINAN), referido a los casos de TB en el escenario pre y post Covid-19 (2016 a 2021 ). Resultados: Según la Organización Mundial de la Salud (OMS), Brasil se encuentra entre los 20 países con mayor incidencia de casos absolutos de TB en el mundo, Pará tiene un promedio anual de 4.166 casos de tuberculosis, estando relacionado con temas de desarrollo social, salud precaria; deficiencia de la gestión pública, que limitan las acciones de prevención, en consecuencia, se ha incrementado el número de casos de la enfermedad. En 2020, hubo una caída del 16\% en la notificación de nuevos casos en comparación con 2019. Estas cifras se explican por las medidas preventivas adoptadas durante la pandemia. Conclusión: El estado de Pará, a pesar de las adversidades sociales y económicas, ha mostrado una reducción en las tasas de TB y el nuevo coronavirus, según los boletines epidemiológicos, esto puede estar vinculado a medidas de distanciamiento social.

Palabras clave: Tuberculosis, COVID-19, Pandemia, Enfermedad respiratoria.

\section{INTRODUÇÃO}

A Tuberculose (TB) é uma doença infectocontagiosa causada pelo Mycobacterium tuberculosis, sendo os pulmões os órgãos mais af etados, porém também pode acometer os rins, a pele, os ossos, os gânglios e vários outros órgãos e tecidos. Além disso, sua forma de transmissão vai ocorre pela via aérea a partir da inalação de aerossóis que contêm os bacilos, os quais são expelidos pela tosse, espirro ou aerossóis durante a fala de doentes com TB pulmonar ou laríngea. Até onde se sabe que o bacilo apresenta capacidade de permanecer no ambiente por um período de até oito horas, mas esse tempo pode se prolonga quando o domicílio não apresenta ventilado e arejado. Por outro lado, somente as pessoas com a forma ativa da doença e bacilíferas são capazes de transmitir a doença (SECRETARIA DE ESTADO DE SAUDE PUPLICA (SESPA), 2021a).

Os primeiros achados históricos foram registros escritos de TB do médico italiano Girolamo Fracastoro em 1546, que relatou as características de contágio da doença. Precisamente, em 24 de março de 1882, o méd ico alemão Robert Koch mostrou à comunidade científica pela primeira vez o isolamento do $M$. tuberculosis, identificando-o como agente etiológico. Em homenagem ao médico, o agente é também conhecido como bacilo de Koch (SANTOS RA, et al., 2019).

De acordo com relatos sobrea TB, já foram encontrados vestígios em restos humanos na era pré-históricos na Alemanha além de relatos a cerca de oito mil anos antes de Cristo (A.C.). Por ser ainda uma doença desconhecida na época, assim como diversas outras, era vista como um castigo divino. Esse pensamento, no entanto, foi desmistificado por Hipócrates, na Grécia em XXX AC. Segundos estudos sé mostrou que a tuberculose poderia ser algo natural e passou a denominá-la de Tísica. A expansão desse mal-estar pelo mundo deu-se com o advento das guerras, que estreitavam o contato entre indivíduos (PEREIRA VMLC, 2020).

Segundo a Organização Mundial da Saúde (OMS), a TB é a principal causa de morte por um único agente infeccioso em todo o planeta, além da principal causa de morte entre pessoas vivendo com Vírus da Imunodeficiência Humana (HIV). Em 2019, cerca de 1,2 milhão de pessoas e outras 10 milhões desenvolveram a doença em todo o mundo (SESPA, 2021b).

Ainda em 2019 foram diagnosticados 73.864 novos casos no Brasil, além de apresentar uma incidência de 36 casos por 100 mil habitantes e a ocorrência de 6.700 óbitos, correspondendo a um coeficiente de mortalidade igual a 3,17 óbitos por 100 mil habitantes. O Brasil consta entre os 20 países com as mais altas incidências por casos absolutos de TB do mundo de TB e TB/HIV. Embora represente um alerta de atenção redobrada, cabe observar que a proporção de casos no Brasil, quando se considera o número de casos por 100 mil habitantes o coloca na $30^{\text {a }}$ posição do ranking (2,4 mortes/100 mil habitantes), bem abaixo da média global (17 mortes/100 mil habitantes) e abaixo de outros países da América Latina, como Peru e Bolívia (OMS, 2018).

Quando se observa os números de casos de TB nas Américas, o Brasil ganha destaque, pois mesmo tendo uma redução nos anos de 2010 e 2016, a taxa de incidência no país aumentou nos anos de 2017 e 
2018 em comparação ao período anterior (SILVA DR, et al., 2021). No Pará, segundo a Secretaria de Estado de Saúde Pública do Pará (SESPA), no ano de 2019 foram registrados os maiores números de novos casos de tuberculose do país, tendo o Pará uma média anual de 4.166 casos novos de TB e uma taxa de incidência média de 48,89\%. Nos últimos 5 anos, esse número vinha apresentando uma tendência de elevação, porém, em 2020, apresentou uma queda de 8,62\% (SESPA, 2021b).

Segundo o Sistema de Informação de Agravos de Notificação (SINAN), todos os casos de TB são notificados somente após confirmação diagnóstica, ou seja, não existe notificação de casos suspeitos. Deverão ser notificados os casos novos, recidivas, outros reingressos e transferências, utilizando a ficha de notificação/investigação de tuberculose (MINISTÉRIO DA SAÚDE, 2019).

Em grandes partes das pessoas, os sintomas da infecção primária podem ser assintomáticos ou brandos, motivar sintomas como febre e dor torácica pleurítica ocasional (GREENE RJ e HARRIS D, 2012). Entretanto, quando a doença fica mais avançada, outros sintomas começam a aparecer, como sudorese noturna, perda ponderal, anorexia, mal-estar e fraqueza. Com a longa duração da doença, os pacientes apresentam tosse com hemoptise maciça ocasional, que é o sintoma mais recorrente, e qualquer pessoa que apresente tosse há mais de três semanas é classificada como sintomático respiratório e deve ser realiza exames para TB (BRASIL, 2009; PINTO MFT, et al., 2017; LONGO DAN L, et al., 2013).

A doença pode ser diagnosticada por meio de história clínica, radiografia de tórax, Tomografia Computadorizada (TC) de tórax, broncoscopia, teste tuberculínico ou reação de Mantoux (SULIS G, et al., 2014; WORLD HEALTH ORGANIZATION (WHO), 2018). O diagnóstico ouro da TB é o exame de baciloscopia de campo claro, que permite a realização de pesquisa de bacilos álcool-ácido resistentes (BAAR) em lâminas de amostra laboratoriais de escarro, onde as amostras podem ser preparadas e coradas pelos métodos padrões como Ziehl-Neelsen ou Kinyoun (SERÃO MKM, 2020).

Porém o diagnóstico da TB durante a pandemia do novo coronavírus deve ser realizado com muita atenção quanto aos aspectos clínicos, pois ambas as moléstias apresentam similaridade como a febre e complicações respiratórias. Além disso, a TB e a COVID-19 podem ser identificadas no paciente simultaneamente. Portanto é fundamental observar com atenção o diag nóstico da COVID-19, através da tomografia computadorizada do tórax, pois é possível também identificar a inf ecção tuberculosa preexistente não diagnosticada. Mediante aos altos índices de tuberculose, deve-se sempre considerar a possibilidade desse diagnó stico codominante para que possa agir de maneira adequada para o tratamento de ambas (SILVA RS, et al., 2021).

Já o tratamento poderá apresenta uma dura no mínimo seis meses, esse tipo de tratamento poderá ser encontrado na forma gratuitamente no Sistema Único de Saúde (SUS). Nesse período, são usados quatro medicamentos, há casos de TB que são necessário a utilização dos medicamentos: rif ampicina, pirazinamida, isoniazida e etambutol. Por outro lado, a enfermidade só possui uma cura quando o tratamento é f eito de forma adequada, até o final de todo o processo (BRASIL, 2017). Ademais, assim como ocorre quando se confirma um caso de TB no país, onde a notificação é obrigatória, dados de cura, falecimento, abandono do tratamento e óbito ocorrido por outras causas durante o tratamento deve ser notificado também (RABAHI FR, et al., 2017)

O novo Coronavirus 2 da Síndrome Respiratória Aguda Grave (SARS-COV-2), popularmente conhecido como vírus da COVID-19, foi identificado como causador da síndrome respiratória aguda grave (WHO, 2021). Embora a COVID-19 comprometa principalmente os pulmões, órgãos como o coração, o fígado, os rins e o cérebro também podem ser acometidos (HUANG C, et al., 2020). A covid-19 já registra a marca de 252.902,685 casos confirmados mundialmente, sendo que nas Américas esses números são de 95.120,017 casos confirmados (ORGANIZAÇÃO PAN-AMERICANA DE SAÚDE (OPAS), 2021). Nesse cenário o Brasil apresenta o quantitativo de 21.953,838 casos confirmados, destes a região Norte registra o número de 1.874.594 casos positivos, sendo que o Pará até a data de 14 de novembro de 2021 apresentou o número de 602.081 casos confirmados (MINISTÉRIO DA SAÚDE, 2021).

No período da pandemia do novo coronavírus, registrou-se uma queda no número de registros da TB, isso pode estar relacionado com as medidas adotadas pela população, como o uso de máscaras, distanciamento 
social, protocolos de higiene como lavar mais as mãos, usar álcool em gel dentre outros, que contribuíram não somente para a proteção contra a covid-19, como contra outras doenças respiratórias. Esses dados também podem estar ligados com a baixa procura da população as unidades básicas de saúde, já que muitas por medo de contrair o covid-19 isolavam-se em casa. Como neste período as unidades de saúde estavam empenhadas em acompanhar casos da covid, outras enfermidades podem ter ficado com subnotificação (MINISTÉRIO DA SAÚDE, 2021).

Outro fator relevante para Grandes estudos de coorte relataram que $90-99 \%$ dos indivíduos infectados com SARS-CoV-2 desenvolvem anticorpos neutralizantes em 2-4 semanas após a infecção. Sendo assim, o objetivo deste estudo foi realizar a comparação do perfil epidemiológico da TB no Estado do Pará, fazendo uma comparação da doença em momentos distintos, antes e após a COVID-19.

\section{MÉTODOS}

Este trabalho trata-se de um estudo epidemiológico observacional descritivo com abordagem quantitativa, realizado por meio do levantamento de dados secundários do SINAN, disponível na plataforma eletrônica do Departamento de Informática do Sistema Único de Saúde (DATASUS) referente a distribuição espacial dos casos de TB no cenário pré e pós Covid-19 (2016 a 2021) (BRASIL, 2021; SOUSA JÚNIOR CP, et al., 2021).

Os dados coletados são referentes ao Estado do Pará, situado no centro Leste da região Norte do Brasil, considerado o segundo do País em superfície, que representa mais de duas vezes o território da França. Limita-se ao norte com o Suriname e o Estado do Amapá; a Nordeste com o Oceano Atlântico; a Leste com os Estados do Maranhão e Tocantins; ao Sul com o Estado de Mato Grosso; e a Noroeste com a Guiana e o Estado de Roraima. Com área territorial total 1.245.870,707 km² com população no censo realizado em 2021 pelo Instituto Brasileiro de Geografia e Estatística (IBGE), apontou um total de 8.777.124 pessoas.

A inclusão dos casos avaliados nesse estudo teve como critérios de escolha apenas os que foram registrados nos municípios do Estado do Pará, durante os anos de 2016 a 2021 e notificados ao SINAN. Já os de exclusão foram os casos notificados fora da região citada ou fora do período analisado pela pesquisa. Dessa maneira, para a análise dos casos considerou-se as variáveis: município, ano, sexo, faixa etária, escolaridade e condições socioeconômicas antes e após a pandemia. Em seguida, os dados foram organizados e tabulados utilizando-se o programa Microsoft Excel 2016, de acordo com as informações obtidas no site do SINAN (SOUSA JÚNIOR CP, et al., 2021).

\section{RESULTADOS E DISCUSSÃO}

A OMS classificou a TB como uma emergência mundial, permanecendo como um problema de saúde pública até os dias atuais, além de ser priorizada em diversos países (BARROS NO, et al., 2019). Sabendo se que a TB ainda é uma das principais preocupações nos serviços de saúde pública no Brasil e que o novo coranavírus tem tomado proporções emergenciais devidos aos inúmeros casos confirmados, fazendo-se necessário planejar maneiras adequadas de tratar ambas as infecções. Tendo em vista que o controle da COVID-19 é similar ao controle da TB no que se refere aos planos de avanço da doença através da sua identificação precoce, profilaxia e monitoração dos pacientes, é possível dessa forma reduzir a disseminação de ambas (MINISTÉRIO DA SAÚDE, 2021).

No ano de 2015 tal acometimento foi responsável pelo maior número de mortes causadas por doenças infecciosas no mundo, chegando até mesmo a ultrapassar os casos de HIV. Esses números foram superados no ano de 2020, quando a COVID-19 acabou por exceder a TB em número de óbitos por dia (SILVA DR, et al., 2021).

A população deste estudo constituiu-se pelos casos registrados nos boletins epidemiológicos de TB notificados ao Sistema de Informações de Agravos de Notificações (SINAN) no período de 2016 a 2020, que residiam nas áreas urbanas e rurais no estado do Pará. A escolha desta região se deu por ser prioritária para o controle dessa doença devido à elevada incidência, à existência de aglomerados habitacionais precários e por apresentar expressiva representatividade de população indígena, considerada pelas políticas públicas como vulnerável (ANDRÉ SR, et al., 2020). 
No Brasil, questões como pobreza, saneamento básico precário, aumento da concentração de pessoas advindas de outros estados para os grandes centros, envelhecimento populacional, gestão deficiente na saúde, todas essas problemáticas corroboram nos processos de diagnóstico, profilaxia e tratamento, dificultando o declínio no número de doenças oriundas pelo contexto social (BARBOSA IR, et al., 2013).

Ademais, pode-se afirmar que a TB é negligenciada, pois os estímulos ao estudo de novos fármacos por parte das indústrias farmacêuticas ainda é pouco, e por parte do Governo não é diferente, já que o investimento em métodos novos de diagnósticos também não é o bastante, tornando a situação ainda pior diante da pandemia da COVID-19 (MACIEL ELN, et al., 2020). Dessa forma, no Brasil, certamente, não alcançaremos o propósito de pôr um fim a TB, com expectativas de redução em $95 \%$ da mortalidade e em $90 \%$ da sua incidência, nos períodos de 2015 a 2035. Para comprovar o estudo, pode-se citar o alerta anunciado pelo Ministério da Saúde sobre dados da tuberculose nos anos de 2019, que mostrou o aumento da incidência da doença no Brasil, demostrado no Gráfico 1 (MACIEL ELN, et al., 2020).

Já o Estado do Pará, segundo o Ministério da Saúde, vem analisando a média nacional e foi constatado que o Brasil consta na $16^{\circ}$ entre os 22 países listados pela OMS, pois apresenta uma das grandes concentrações da carga mundial de tuberculose é de $80 \%$ dos casos. No ano de 2013 , foram registrados em torno de 73 mil casos novos da doença em todo o país (SINDICATO DOS MÉDICOS DO PARÁ (SINDIMEPA), 2021). Em virtude de a Região Norte apresentar uma das maiores taxas de tuberculose, torna-se importante a realização de trabalhos de cunho epidemiológico voltado a esta região para que, desta maneira, se torne mais compreensível a distribuição da doença nesta região e suas peculiaridades em relação a outras regiões do Brasil (PEREIRA LFS, et al., 2019). Entre os anos de 2016 a 2020, por exemplo, foram notificados aproximadamente 23.859 casos de TB nos municípios do Pará, distribuídos nos 144 municípios, segundo o DATASUS.

O Programa de Controle da Tuberculose (PCT) encontra-se implantado em todos os 144 município do estado, com ações voltadas para o diagnóstico, vem monitorando o tratamento dos casos já registrado e tomando medidas de prevenções em grupos de maior vulnerabilidade a TB. Anualmente, a coordenação acompanha in loco as regionais de saúde, assim como rotineiramente realiza monitoramento indireto através dos sistemas de informação e reuniões virtuais, considerando o cenário atual (MINISTÉRIO DA SAÚDE, 2021).

Em 2019, segundo o Ministério da Saúde, o Estado destacou-se no país com o maior percentual de casos novos de TB notificados e acompanhados na Atenção Primária à Saúde - 93\%, resultado da articulação com esses serviços, como mostra a Gráfico 1 (MINISTÉRIO DA SAÚDE, 2021).

Gráfico 1 - Coeficiente de incidência de tuberculose geral (por 100 mil hab.) e taxa de incidência de casos de tuberculose no Pará, 2016 a 2020. Brasil, 2016 a 2020.

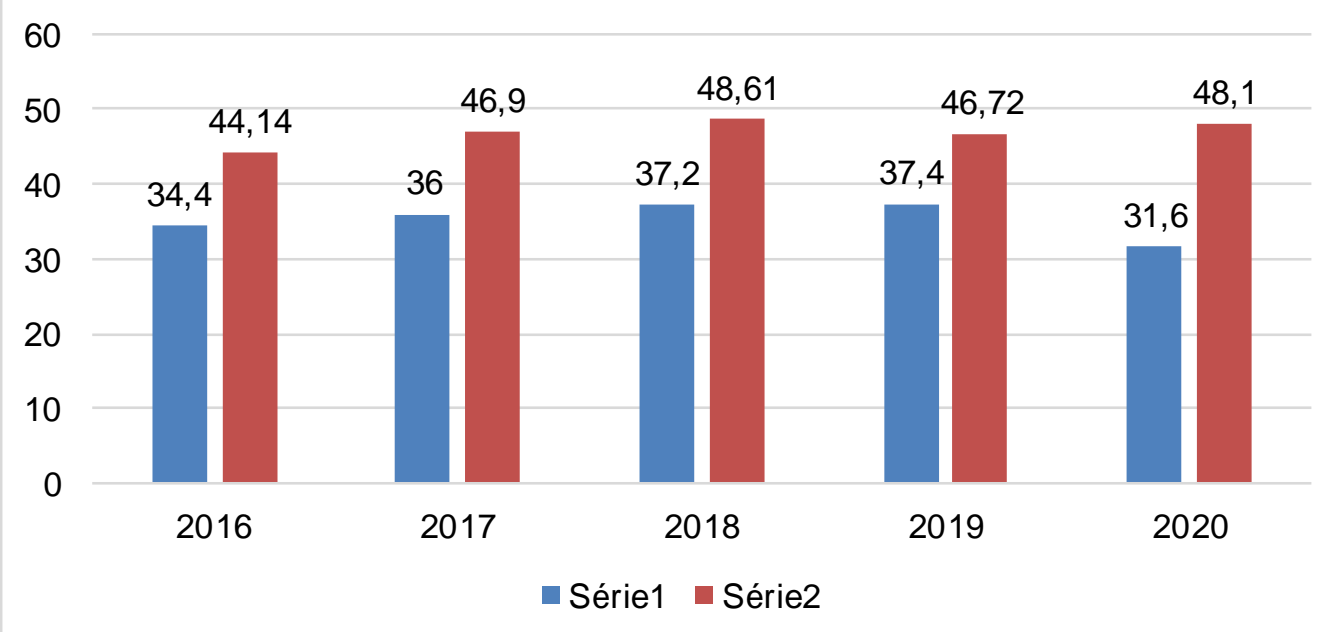

Fonte: Amaral ACC, et al., 2021; dados extraídos do SINAN/SESPA/DVS e dos Estaduais de Saúde/Ministério da Saúde; Instituto Brasileiro de Geografia e Estatística, 2021. Dados atualizados em 2021 e sujeitos à revisão. 
O Pará apresenta uma média anual de 4.166 casos novos de tuberculose e uma taxa de incidência média de $48,89 \%$. Nos últimos 5 anos, essa taxa de incidência vinha apresentando uma tendência de elevação, porém em 2020 apresentou uma queda de 8,62\%. Pode-se inferir que esta queda esteja associada à pandemia causada pelo coronavírus, que limitou o acesso das pessoas aos serviços de saúde, assim como também diminuiu as atividades desenvolvidas pelos profissionais de saúde, que trabalham na linha de frente para o controle da tuberculose, seja na esfera municipal ou estadual, como busca de sintomáticos respiratórios, devido à necessidade do distanciamento social. Entretanto, é possível que passado esse período, o número de casos de tuberculose volte a subir (MINISTÉRIO DA SAÚDE, 2021).

No ano 2019, antes do ocorrido da pandemia, foram notificados 4.532 óbitos ocasionados pela doença, apresenta uma taxa de mortalidade de 2,2 óbitos/100 mil habitantes, apresentando os dados iguais de dois anos anteriores. Porém, desde 2010, os números anual de óbitos por TB no Brasil vêm se variado de 4.400 a 4.600, já os números de mortalidade, de 2,3 a 2,2 óbitos por 100 mil habitantes (MINISTÉRIO DA SAÚDE, 2019).

Ainda em 2019, 13 unidades federativas demonstraram coeficiente de mortalidade por TB aproximado ou superior aos números de coeficiente do país, que representam: Amazonas, Rio de Janeiro, Pernambuco, Rio Grande do Sul, Pará, Maranhão, Rio Grande do Norte, Roraima, Ceará, Acre, Alagoas Bahia e Espírito Santo. As metrópoles desses estados relacionados também mostraram os maiores coeficientes de mortalidade quando comparadas às demais capitais do país.

Os casos em ambos os sexos, sendo possível analisar que a ocorrência é maior entre os homens, com uma média de 1.580 a mais de casos confirmados (MINISTÉRIO DA SAÚDE, 2021).

No Pará, a média dos casos de TB de acordo com o sexo contínua semelhante a incidência nacional e o sexo masculino continua a ser o mais acometido. Acredita-se que isso esteja associado ao estilo de vida, como abuso de álcool, drogas, tabagismo, exposição sexual que podem interferir na imunidade, predispondo ao adoecimento por tuberculose como mostra o Gráfico 2 (SILVA GLA, et al., 2021).

Gráfico 2 - Casos confirmados por ano diagnóstico segundo sexo, Período: 2016 - 2020.

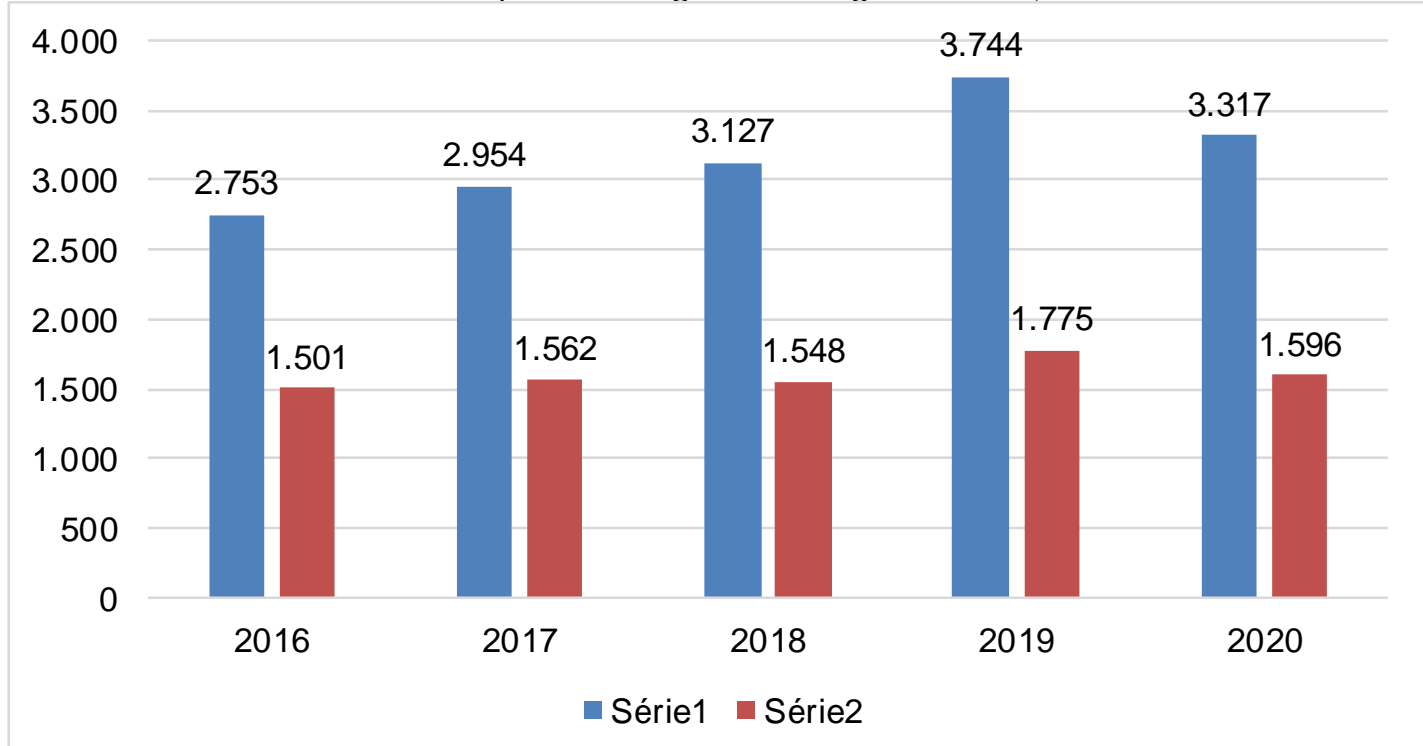

Fonte: Amaral ACC, et al., 2021; dados extraídos do Ministério da saúde/SVS, Sistema de Informação de Agravos de Notificação, Sinan Net, 2021.

Na Tabela 1 podemos observar o número de casos notificados de TB nos anos de 2016 a 2020 por faixa etária no Estado do Pará, revelando que a idade entre 25 e 34 ano s foi a mais acometida no período de 2016 a 2019, com diminuição no ano de 2020. Já a faixa etária menos afetada foi a de 0 a 14 anos, como demonstrado na Tabela 1. 
Tabela 1 - Casos registrados da TB segundo a faixa etária.

\begin{tabular}{cccccccc}
\hline Ano & 0 a 14 & 15 a 24 & 25 a 34 & 35 a 44 & 45 a 54 & 55 a 64 & 65 \\
\hline $\mathbf{2 0 1 6}$ & 139 & 879 & 981 & 755 & 605 & 480 & 415 \\
$\mathbf{2 0 1 7}$ & 153 & 946 & 1.023 & 790 & 629 & 517 & 458 \\
$\mathbf{2 0 1 8}$ & 161 & 954 & 1.150 & 817 & 623 & 520 & 450 \\
$\mathbf{2 0 1 9}$ & 199 & 1.183 & 1.344 & 954 & 727 & 584 & 528 \\
$\mathbf{2 0 2 0}$ & 133 & 983 & 1.223 & 897 & 696 & 532 & 449 \\
\hline
\end{tabular}

Fonte: Amaral ACC, et al., 2021; dados extraídos do Ministério da saúde/SVS, Sistema de Informação de Agravos de Notificação, Sinan Net, 2021.

Dos 144 municípios paraenses, podemos obter o número total de casos registrados de TB nos anos de 2016 a 2020. O menor número foi apontado no ano de 2016 e a maior quantidade de acometidos foi em 2019. Segundo um dos diretores da OMS, Tedros Adhanom Ghebreyesus, a responsabilidade pelos líderes globais para a erradicação da TB como problema de saúde pública até 2030 e essa pausa dos serviços essenciais para pessoas com TB apenas representam como a pandemia está prejudicando desproporcionalmente alguns cidadãos mais desprovidos do mundo, que já correm mais risco de manifesta a TB, (ORGANIZAÇÃO DAS NAÇÕES UNIDAS (ONU), 2020). Como mostra o Gráfico 3.

Gráfico 3 - Casos confirmados, Período: 2016 - 2020.

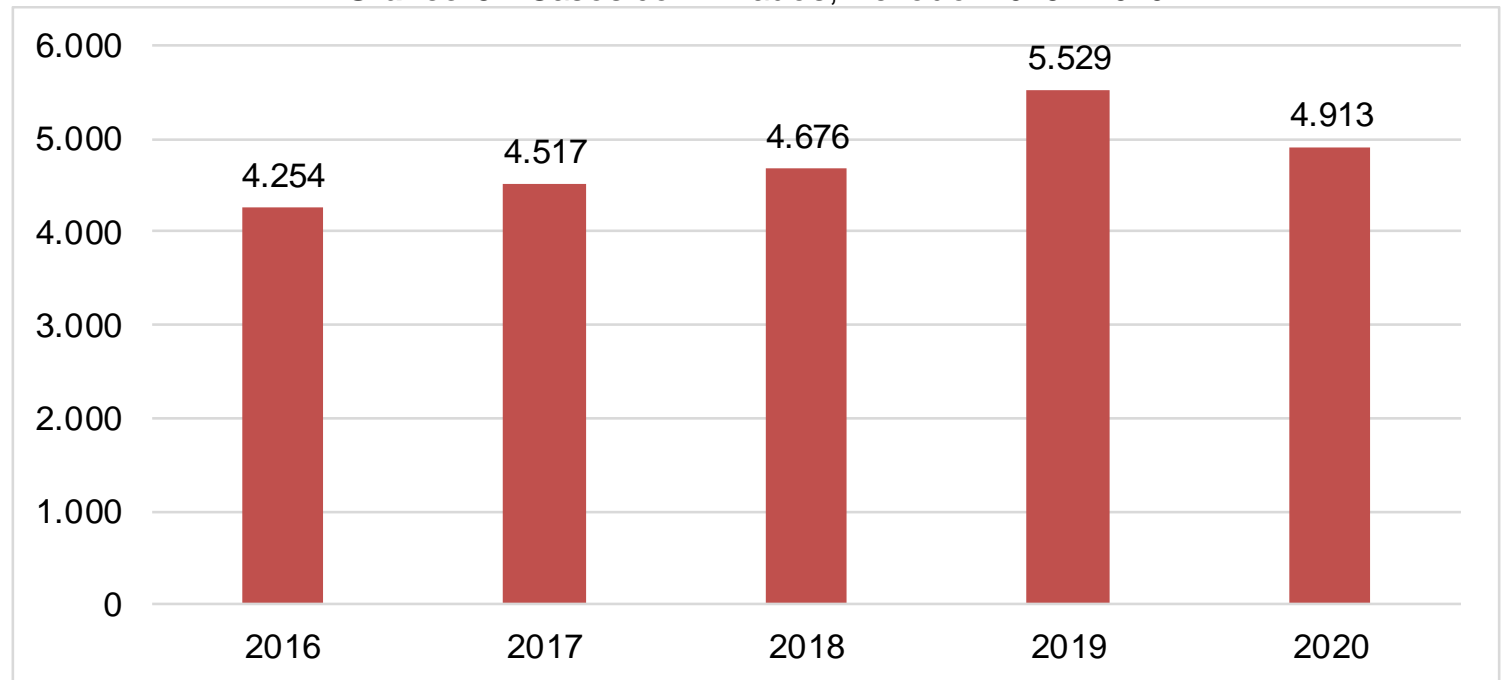

Fonte: Amaral ACC, et al., 2021. Dados extraídos do Ministério da saúde/SVS - Sistema de Informação de Agravos de Notificação (Sinan Net), 2021.

No ano de 2020, apresentou-se uma baixa de $16 \%$ das notificações de casos novos de TB em comparado em 2019. Essa redução foi mais notada no período de abril, sendo que no mês de maio foi possível verificar a maior variação do período (-34\%) em relação às notificados. Paralelamente, foi requisitada uma redução de $14 \%$ no consumo de todos os cartuchos de teste rápido molecular para tuberculose (TRM-TB) quando foi verificado no ano de 2020 ao de 2019, estima-se que medidas preventivas de etiqueta respiratória, como o uso de máscaras obrigatórias pela população para ser prevenir a COVID -19 poderiam ter contribuído para as reduções das transmissões da TB e até podendo interferir em outras infecções respiratórias na comunidade. No entanto, ainda não possui dados corroborantes (OPAS, 2021).

Nos anos de 2015 a 2020, aprestou-se um elevado nos números de casos de TB em populações mais suscetível a esse tipo de doença. Ao longo desse período das decorrências de casos na População Privada de Liberdade (PPL), foi de 5.860 a 8.978; em profissionais de saúde (PS), foi de 837 a 1.043; em estrangeiros, foi de 335 a 542; e na População em Situação de Rua (PSR), foi de 1689 a 2.071, como mostra no Gráfico 4 (MINISTÉRIO DA SAÚDE, 2021). 
Gráfico 4 - Proporção de casos novos de tuberculose diagnosticados em populações vulneráveis no Brasil, de 2015 a 2020.

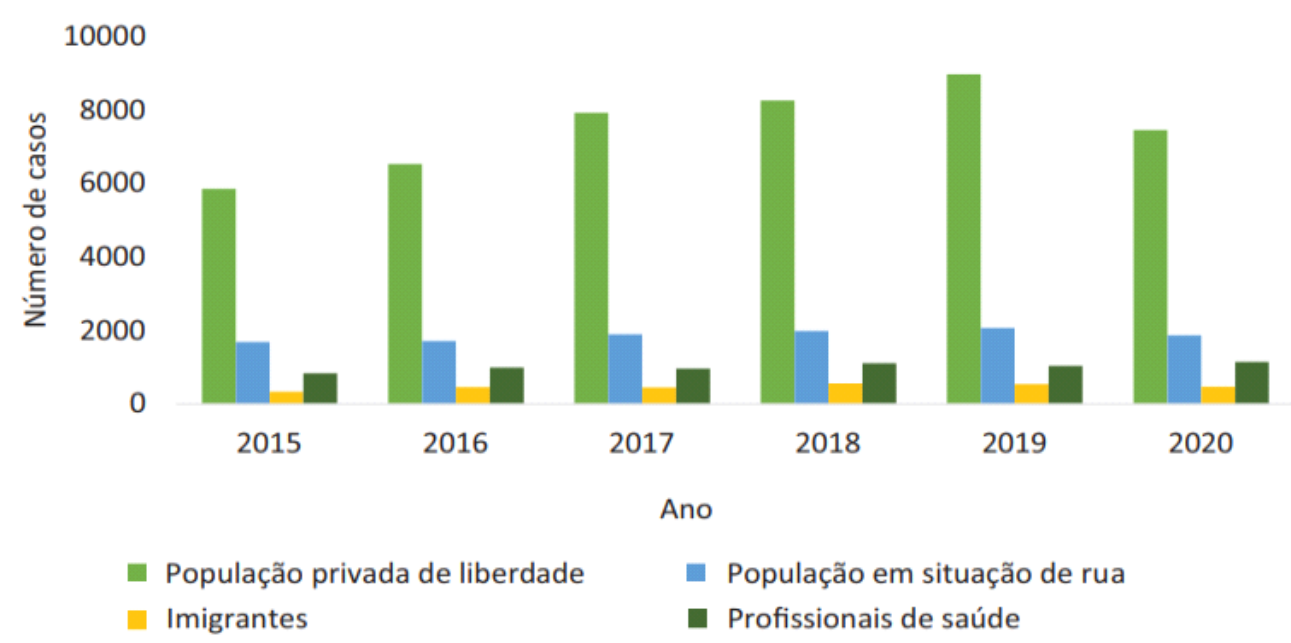

Fonte: Amaral ACC, et al., 2021. Dados extraídos do Sistema de Informação de Agravos de Notificação/Secretarias Estaduais de Saúde/Ministério da Saúde, 2021.

\section{CONSIDERAÇÕES FINAIS}

A pesquisa desse artigo esteve voltada para o levantamento epidemiológico das doenças TB e COVID 19, permitindo identificar que a precariedade em diversas esferas sociais enfrentadas pela população paraense e dos demais estados, estão intrinsecamente envolvidas nas etapas de controle ao avanço de ambas e na sua identificação. Porém observamos também que gradativamente é possível perceber uma diminuição nos casos devidos algumas medidas que devem ainda permanecer, como a melhoria nos hábitos de higiene, ingestão de suplementos e uso de máscara. Sendo assim as informações abordadas neste trabalho, é imprescindível para avaliação do cenário atual da tuberculose no estado do Pará e para possíveis medidas de controle desta enfermidade por parte dos órgãos governamentais.

\section{REFERÊNCIAS}

1. ANDRÉ SR, et al. Tuberculose associada às condições de vida em município endêmico da região Norte do Brasil. Rev. Latino-Am. Enfermagem, 2020;28: 33-43.

2. BARBOSA IR, et al. Análise da distribuição espacial da tuberculose na região Nordeste do Brasil, 2005-2010. Rev. Epidemiol. Serv. Saúde, 2013;22(4). 687-695.

3. BARROS NO, et al. Avaliação Epidemiológica dos Casos de Tuberculose da Região Nordeste do Brasil, no período de 2010 a 2019. Centro Universitário Tiradentes (Unit), 2021.

4. BRASIL. Cartilha para o Agente Comunitário de Saúde: tuberculose. 2017. Disponível em: https://antigo.saude.gov.br/images/pdf/2017/julho/03/cartilha_acs_tb_11jun17_site_100ex.pdf. Acessado em: 24 de agosto de 2021.

5. BRASIL. Portal do Domínio Público. Biblioteca Digital desenvolvida em software livre. O estado do Pará. Disponível em: http://www.dominiopublico.gov.br/download/texto/m re000008.pdf. Acessado: em 26 de agosto de 2021.

6. GREENE RJ, HARRIS ND. Patologia e Terapêuticas para Farmacêuticos: Bases para a Prática da Farmácia Clínica. 3aㅡ ed. Porto Alegre: Artmed, 2012; 432-433.

7. HUANG C, et al. Clinical features of patients infected with 2019 novel coronavirus in Wuhan, China. The Lancet, 2020; 395(5): 497-506.

8. INSTITUTO BRASILEIRO DE GEOGRAFIA E ESTATÍSTICA (IBGE). Panorama - Pará. Disponível em: https://cidades.ibge.gov.br/brasil/pa/panorama. Acessado em:26 de agosto de 2021.

9. JÚNIOR AMF, et al. Análise da incidência da tuberculose nos estados da região norte do Brasil. Revista Eletrônica Acervo Científico, 2021;24;1-7.

10. LONGO DAN, et al. Manual de medicina de Harrison. 18ª ed. Porto Alegre: Artmed, 2013.

11. MACIEL ELN, et al. Tuberculose e coronavírus: O que sabemos?. Rev. Epidemiol. Serv. Saúde, 2020; $29(2): 128$.

12. MINISTÉRIO DA SAÚDE. Boletim Epidemiológico de tuberculose. 2021. Disponível em: https://www.gov.br/saude/pt$\mathrm{br} / \mathrm{media} / \mathrm{pdf} / 2021 / \mathrm{marco} / 24 / \mathrm{boletim}$-tuberculose-2021_24.03. Acessado em:30 de ago de 2021. 
13. MINISTÉRIO DA SAÚDE. Secretaria de Vigilância Em Saúde. Boletim Epidemiológico. Brasil Livre da Tuberculose: evolução dos cenários epidemiológicos e operacionais da doença. 2019. Disponível em: https://portalarquivos2.saude.gov.br/images/pdf/2019/marco/22/2019-009.pdf. Acessado em:30 de agosto de 2021.

14. MINISTÉRIO DA SAÚDE. Secretaria de Vigilância Em Saúde. Boletim Epidemiológico da Tuberculose, número especial. 2021. Disponível em: https://www.gov.br/saude/pt-br/media/pdf/2021/marco/24/boletim-tuberculose2021_24.03. Acessado em: 30 de agosto de 2021.

15. MINISTÉRIO DA SAÚDE. Secretaria de Vigilância em Saúde. Boletim Epidemiológico Especial Doença pelo Coronavirus Covid 19, semana epidemiológica. 2021. Disponível em: https://www.gov.br/saude/pt- HYPERLINK "https://www.gov.br/saude/pt-

br/media/pdf/2021/marco/05/boletim_epidemiologico_covid_52_final2.pdf.\%20Acessado"br/media/pdf/202 1/marco/0 5/boletim_epidemiologico_covid_52 final2.pdf. Acessado em:15 de novembro de 2021.

16. ORGANIZAÇÃO DAS NAÇÕES UNIDAS (ONU). Progresso global no combate à tuberculose está em risco, afima OMS. 2020. Disponível em: https://brasil.un.org/pt-br/95855-progresso-global-no-combate-tuberculose-esta-emrisco-afirma-oms. Acessado em: 13 de janeiro de 2022.

17. ORGANIZAÇÃO PAN-AMERICANA DA SAÚDE (OPAS). Diagnóstico de novos casos de tuberculose caiu entre $15 \%$ e 20\% nas Américas em 2020 devido à pandemia. 2021. Disponível em: https://www.paho.org/pt/noticias/24-3-2021diagnostico-novos-casos-tuberculose-caiu-entre-15-e-20-nas-americas-em-2020. Acessado em: 14 de setembro de 2021.

18. ORGANIZAÇÃO PAN-AMERICANA DA SAÚDE (OPAS). Washington, WHO Coronavirus (COVID-19) Dashboard. 2021. Disponível em: https://covid19.who.int. Acessado em:13 de novembro De 2021.

19. ORGANIZAÇÃO MUNDIAL DA SAÚDE. Global Tuberculosis Report 2018.

20. PEREIRA LFS, et al. Epidemiologia da tuberculose no estado do Pará. Braz. J. Hea., 2018;2(2): 800-808.

21. PEREIRA VMLC. Mortalidade por tuberculose no hospital raoulfollereau em bissau e fatores associados. Dissertação (Mestrado em Estatística para a Saúde) - Universidade Nova de Lisboa Instituto de Higiene e Medicina Tropical, Lisboa, 2020;58p.

22. PINTO MFT, et al. Budget impact of the incorporation of GeneXpertMTB/RIF for diagnosis of pulmonary tuberculosis from the perspective of the Brazilian Unified National Health System, Brazil, 2013-2017. Cad Saude Publica, 2017; 33(9): e00214515.

23. RABAHI MF, et al. Tratamento da tuberculose. Rev. Jornal Brasileiro de Pneumologia, 2017; 43(5): 472-486.

24. SANTOS RA, et al. Educação em Saúde: ações de prevenção da tuberculose em instituições de ensino na cidade de Manaus, Amazonas. Nexus, Revista de Extensão do IFAM, 2019;9(5).

25. SECRETARIA DE ESTADO DE SAÚDE PÚBLICA (SESPA). Boletim epidemiológico da tuberculose. 2021 b. Disponível em: http://www.saude.pa.gov.br/wp-content/uploads/2021/07/BOLETIM-EPIDEMIOLOGICO-DATUBERCULOSE-1.pdf. Acessado em:24 de agosto de 2021.

26. SECRETARIA DE ESTADO DE SAÚDE PÚPLICA (SESPA). Diretoria de Vigilância em Saúde, Coordenação Estadual do Programa de Controle da Tuberculose - Plano Estadual de Tuberculose. 2021a. Disponível em: http://www.saude.pa.gov.br/wp-content/uploads/2021/07/BOLETIM-EPIDEMIOLOGICO-DA-TUBERCULOSE-1.pdf. Acesso em:24 de agosto de 2021.

27. SERÃO MKM. Detecção automática de bacilos em baciloscopia de campo claro usando aprendizado profundo e técnica de imagem mosaica. Dissertação (Mestrado em Engenharia Elétrica) - Universidade Federal do Amazonas, Manaus, 2020; 82p.

28. SILVA DR, et al. Tuberculose COVID-19, o novo dueto maldito: quais as diferenças entre Brasil e Europa?. Rev. Jornal Brasileiro de Pneumologia, 2021;47(2):1-8.

29. SILVA GLA, et al. Boletim Epidemiológico da Tuberculose. Secretaria de estado de saúde pública, 2021;1:17p. Abril de 2021.

30. SILVA RS, et al. Tuberculose e COVID-19, o novo dueto maldito: quais as diferenças entre Brasil e Europa?. Rev. Jornal Brasileiro de Pneumologia, 2021;47(2): e20210044.

31. SINDICATO DOS MÉDICOS DO PARÁ (SINDIMEPA). Pará lidera em casos de Tuberculose. Disponível em: https://sindmepa.org.br/para-lidera-em-casos-de-tuberculose/. Acessado: em 31 de agosto de 2021.

32. SOCIEDADE BRASILEIRA DE PNEUMOLOGIA E TISIOLOGIA. Ministério da Saúde (Org.). III Diretrizes para Tuberculose da Sociedade Brasileira de Pneumologiae Tisiologia. Jornal Brasileiro de Pneumologia. 2009. Disponível em:http://www.scielo.br/pdf/jbpneu/v35n10/v35n10a11.pdf. Acessado em:2 de setembro. 2021.

33. SOUSA JÚNIOR CP, et al. Análise epidemiológica dos casos de tuberculose na Região de Integração do Lago de Tucuruí - PA. Rev. Research, Society and Devlopment, 2021;10(6):1-11.

34. SULIS G, et al. Tuberculosis: epidemiology and control. Rev. Mediterranean Journal of Hematology and Infectious Diseases, 2014;6(1): 1-8.

35. WORLD HEALTH ORGANIZATION (WHO). 2021. In: WHO Coronavirus (COVID-19) Dashboard. Disponível em: https://covid19.who.int/ . Acessado em: 2 de setembro de 2021.

36. WORLD HEALTH ORGANIZATION (WHO). 2021. Global health TB report. Disponível em: https://www.who.int/tb/publications/global_report/en/.Acessado em:2 de setembro de 2021. 